\title{
Correction to: Proceedings of GeoShanghai 2018 International Conference: Multi-physics Processes in Soil Mechanics and Advances in Geotechnical Testing
}

Liangbo Hu, Xiaoqiang Gu, Junliang Tao, and Annan Zhou

\section{Correction to:}

L. Hu et al. (Eds.): Proceedings of GeoShanghai 2018

International Conference: Multi-physics Processes in Soil Mechanics and Advances in Geotechnical Testing, GSIC 2018, https://doi.org/10.1007/978-981-13-0095-0

In the original version of the book, the incorrect second author name "We-Wei Du" has to be corrected as "Wei-Wei Du" in the chapter "Model Test of Seepage Erosion Around Defective Buried Pipelines", frontmatter and backmatter. The erratum book has been updated with the change.

In the original version of the book, an incorrect image was labelled as Figure 5 in Chapter 50, which has now been corrected. The book and the chapter have been updated with the change.

The updated version of these chapters can be found at https://doi.org/10.1007/978-981-13-0095-0_30

https://doi.org/10.1007/978-981-13-0095-0_50 